\title{
EFFECT OF MINDFULNESS MEDITATION AND COPING STRATEGIES ON AFFECT AND DEPRESSION SYMPTOMATOLOGY AMONG MEDICAL STUDENTS DURING NATIONAL LOCKDOWN - A PROSPECTIVE, NON-RANDOMISED CONTROLLED TRIAL
}

\author{
Thomas Khan-White \\ Faculty of Medicine, Imperial College London (UK)
}

\begin{abstract}
This prospective, non-randomised controlled trial aimed to investigate the effects of brief app-based sessions of mindfulness meditation (MM) and utilisation of either problem-focused (PFC) or emotion-focused coping (EFC) styles on positive affect (PA), negative affect (NA) and depression symptomatology during nationwide lockdown due to coronavirus disease 2019 (COVID-19). This study enrolled 19 medical students to undergo a 10-minute MM intervention. These same students were also divided into either PFC or EFC groups. Affect was measured using the positive and negative affect schedule (PANAS), depression symptomatology was measured using the centre for epidemiological studies-depression (CES-D) scale and the brief COPE survey was used to categorise individuals as either PFC or EFC. Analysis showed no significant between-subject interaction for MM on PA, NA or CES-D score. Analysis of coping styles showed no significant between-subject interactions in relation to PA or NA, though CES-D scores approached significance $(p=0.057)$ and PFC scored significantly lower than EFC at baseline $(p=0.02)$. Significant within-subject effect of time found for PA and NA across both analyses $(p<0.05) .61 .1 \%$ of cohort scored above CES-D cut-off for being considered at risk of depression. These results identify a need for further work into more intensive MM interventions with larger sample sizes as well as study into stressors associated with lockdown to more fully establish whether PFC strategies are the most beneficial. In addition, a clearly high level of psychological distress has been implicated in this study population, which may have implications for student pastoral care during this period.
\end{abstract}

Keywords: COVID-19, mindfulness, coping strategies, depression, affect.

\section{Introduction}

Whilst widespread quarantine measures have proven invaluable in slowing the global spread of COVID-19, they do also present unique challenges. It is well-documented in the literature that quarantine can have detrimental psychological impacts and can worsen pre-existing mental illness (Hawryluck et al.,2004). Therefore, the challenges to come demand an intervention that is widely accessible to the population. Online mindfulness meditation (MM) has been shown in to be effective in reducing stress, irritability, depression and anxiety (Khoury et al.,2015). One population group that has received less focus in the literature is medical students. The interplay of coping strategies may also be an area of relevance. Two broad classifications of coping, problem-focused (PFC) and emotion-focused coping (EFC) have been identified, and their role along with the potential benefit of MM interventions among a cohort of medical students during national lockdown is the subject of this study.

\section{Objectives \& hypotheses}

This study aims to meet the need for research into widely-accessible mental health interventions during the current pandemic and proposes to investigate the effects of a brief MM intervention using Headspace on daily affect and symptoms of depression amongst medical students during lockdown. In addition, this study shall also seek to explore the ability of PFC and EFC styles to predict psychological outcomes of affect and depression symptomatology.

Hypothesis $1\left(\mathrm{H}_{1}\right)$ - The meditation intervention group will experience improved affect and a lower frequency of symptoms associated with depression. 
Hypothesis $2\left(\mathrm{H}_{2}\right)$ - Coping style (PFC or EFC) will predict affect and frequency of symptoms associated with depression.

\section{Methods}

\subsection{Participants}

After applying inclusion and exclusion criteria, 19 medical students were assigned to either a MM intervention or passive control group. Additionally, daily brief COPE survey scores were used to allocate individuals to either PFC or EFC groups.

\subsection{Procedure}

This study was granted ethical approval by the Imperial College Joint Research Compliance Office. All study subjects were asked by email to read through a participant information sheet and then to complete a consent form. Upon receipt of consent forms, participant numbers were issued to preserve anonymity. Baseline questionnaires (Day 1) were then administered. The following day once all baseline questionnaires had been completed the first daily questionnaires were administered along with the first weekly questionnaires. Daily questionnaires were administered every day up until and including Day 16 and weekly questionnaires were administered on Day 2, Day 9 and Day 15. This study ran for a period of 16 days. All questionnaires were conducted online using Qualtrics.

\subsection{Intervention \& control}

The intervention consisted of a daily group video call via Microsoft Teams using 10-minute sessions from Headspace's "basics" section. The control group were then instructed not to practice MM during the study period. 9 individuals were allocated to MM intervention and 10 to control group.

\subsection{Measures}

Affect was assessed using the Positive and Negative Affect Scale (PANAS) and was issued in the baseline survey and on each daily survey. Scores for PA and NA were then summed and averaged to give group daily mean scores. The Brief COPE survey was used to assess each individual's daily coping strategies and was issued in the daily surveys. 9 individuals were allocated to EFC and 10 to PFC groups. Centre for Epidemiological Studies Depression Scale (CES-D) scale issued in weekly questionnaires and used to assess presence and frequency of depressive symptoms.

\subsection{Statistical analysis plan}

All statistical analyses were conducted using SPSS Version 21. A p-value of less than 0.05 was considered significant.

\section{Results}

H1 $_{1}$ - Baseline independent t-tests revealed no significant differences in any of the measures (PA, NA or CES-D) at baseline. No between-subjects effects observed for PA, NA or CES-D scores. Significant within-subject effect of time was observed for both PA and NA (figure 1), with scores generally decreasing over time.

Table 1. Shows p-values for between-subjects (MM intervention) and within-subjects effects (time) for all three study measures.

\begin{tabular}{|c|c|c|}
\hline \multirow{2}{*}{ Measure } & $\begin{array}{c}\text { Between-subjects effects (MM } \\
\text { intervention) }\end{array}$ & Within-subjects effects (Time) \\
\cline { 2 - 3 } & P-value $(\mathrm{p})$ & P-value (p) \\
\hline Positive Affect & 0.997 & $0.002^{*}$ \\
\hline Negative Affect & 0.358 & $0.036^{*}$ \\
\hline CES-D & 0.399 & 0.995 \\
\hline
\end{tabular}

$\mathbf{H}_{2}$ - Baseline independent T-tests revealed no significant differences between EFC and PFC groups on PA and NA measures. However, significant baseline difference was observed between EFC and PFC groups for CES-D score $(\mathrm{p}=0.02 *)$, with PFCs scoring a mean of $14.8 \pm 6.5$ and EFCs scoring a mean of 24.3 \pm 9.1 . No significant between-subject effects of coping style observed for PA, NA or CES-D scores, however CES-D scores approached significance $(p=0.057)$. Significant within-subject effect of time was observed for both PA and NA (figure 2), with scores generally decreasing over time. 
Table 2. Shows p-values for between-subjects (coping style) and within-subjects effects (time) for all three study measures.

\begin{tabular}{|c|c|c|}
\hline \multirow{2}{*}{ Measure } & $\begin{array}{c}\text { Between-subjects effects (Coping } \\
\text { style) }\end{array}$ & Within-subjects effects (Time) \\
\cline { 2 - 3 } & P value $(\mathrm{p})$ & P value (p) \\
\hline Positive Affect & 0.952 & $0.001^{*}$ \\
\hline Negative Affect & 0.104 & $0.034^{*}$ \\
\hline CES-D & 0.057 & 0.994 \\
\hline
\end{tabular}

\section{Discussion}

H1 $_{1}$ - Hypothesis 1 rejected and null hypothesis accepted. Lack of significant between-subject effects for all measures (PA, NA and CES-D) between meditation and control group could relate to the intensity of the MM intervention, as this study used only 10-minute sessions over a two-week period. Other studies demonstrating significance between affect and MM usually use significantly longer and more intense guided MM interventions typically with larger study cohorts (Sears et al.,2009).

$\mathbf{H}_{2}$ - Hypothesis 2 rejected and null hypothesis accepted. No significant between-subject effects of coping style on PA, NA or CES-D during study period. T-testing revealed significant difference at baseline in CES-D scores, with PFCs scoring significantly lower than EFCs. These findings align with those found in isolated, confined and extreme (ICE) environments such as polar or submarine settings, where PFC strategies are often the most commonly used and are most effective in dealing with stressors (Sandal et al.,2003). Given the parallels of quarantine with ICE environments, it may be appropriate to consider methods for dealing with isolation used by those operating in ICE settings.

Within-subject effects - Significant main effect of time observed for PA and NA scores in both $\mathrm{H}_{1}$ and $\mathrm{H}_{2}$ analyses. In both analyses, scores for PA and NA generally decreased in both groups. This may have occurred due to affective blunting, which has been identified as a depressive symptom (Loas et al., 1994). This is supported by relatively high group mean CES-D scores by the same cohorts over the study period.

Other effects - Notable are the relatively high scores on CES-D surveys amongst this cohort, with $61.1 \%$ scoring above cut-off to be considered at-risk for clinical depression. As surveys were completed under condition of anonymity, diagnostic interviews were not possible as part of any follow-up. This finding has implications for student pastoral care during the pandemic.

\section{Conclusion}

The findings of the present study suggest that brief, app-based MM conducted via video conferencing is not effective in influencing affect or depression symptomatology in medical students. In addition, despite significant baseline differences, it cannot be said definitively that coping style influenced these variables either. However, these findings do justify further work with ideally larger sample sizes to more definitively determine whether different coping styles or MM help in weathering lockdown. What the present study has identified however is a clear psychological burden on medical students as evidenced by the high CES-D scores in all groups. It is clear therefore that easily accessible interventions that do not violate the rules of lockdown are required to meet the clear mental health demands the current pandemic places on this population.

\section{References}

Hawryluck L, Gold WL, Robinson S, Pogorski S, Galea S, Styra R. SARS control and psychological effects of quarantine, Toronto, Canada. Emerging Infectious Diseases. 2004 Jul;10(7):1206.

Khoury B, Sharma M, Rush SE, Fournier C. Mindfulness-based stress reduction for healthy individuals: A meta-analysis. Journal of psychosomatic research. 2015 Jun 1;78(6):519-28.

Loas G, Salinas E, Pierson A, Guelfi JD, Samuel-Lajeunesse B. Anhedonia and blunted affect in major depressive disorder. Comprehensive psychiatry. 1994 Sep 1;35(5):366-72.

Sandal GM, Endresen IM, Vaernes R, Ursin H. Personality and coping strategies during submarine missions. Journal of Human Performance in Extreme Environments. 2003;7(1):5.

Sears S, Kraus S. I think therefore I om: Cognitive distortions and coping style as mediators for the effects of mindfulness meditation on anxiety, positive and negative affect, and hope. Journal of clinical psychology. 2009 Jun;65(6):561-73. 